\title{
SMOOTH STRUCTURES ON SPHERE BUNDLES OVER SPHERES
}

\author{
SAMUEL OMOLOYE AJALA \\ Institute for Advanced Study \\ School of Mathematics \\ Princeton, New Jersey 08543 \\ USA \\ and \\ Department of Mathematics \\ University of Lagos \\ Akoka - Yaba \\ Lagos - Nigeria \\ West Africa
}

(Received May 30, 1986)

ABSTRACT. In [1] R. De Sapio gave a classification of smooth structures of a p-sphere bundle over a $q$-sphere with one cross-section and $p<q$. In [2] J. Munkres also gave a classification up to concordance of differential structures in the case where the bundle has at least two cross-sections. In [3] R. Schultz gave a classification in the case $p \geq q$. Here we will give a classification of the $p$-sphere bundle over a $q$-sphere without any cross-section and $p<q$.

KEY WORDS AND PHRASES. Smooth structures, differential classification, internal groups. 1980 AMS SUBJECT CLASSIFICATION CODES: $57 R 55$.

\section{INTRODUCTION}

Let $E$ represent $p$-sphere bundle over a $q$-sphere with $\beta \varepsilon \pi_{q-1} S O(p+1)$ the characteristic class of the corresponding p+1-disc bundle over the q-sphere. In [4] R. De Sapio gave a complete classification of the special case where $\beta=0$. In [5] and [6] Kawakubo and Schultz respectively also gave a classification of $E$ for this special case. This author in [7] gave a generalization of this special case to product of three ordinary spheres. In [1] a classification of $E$ was given for $p<q-1$ and where $E$ has a cross-section and $\beta \neq 0$. In [3] Schultz gave a classification of $E$ for $p \geq q$ and $E$ is without cross-section. We shall here remove the fact that $E$ has a cross-section so that not every element of $\pi_{q-1} S O(p+1)$ can be pulled back to the element $\pi_{q-1} S O(p)$ in the homomorphism $S_{*}: \pi_{q-1} S O(p) \rightarrow \pi_{q-1} S O(p+1)$ induced by the inclusion $s: S O(p) \rightarrow S O(p+1)$. $s^{n}$ denotes the unit $n$-sphere with the usual differential structure in the Euclidean 
$(n+1)$-space $R^{n+1} \cdot \Sigma^{n}$ denotes an homotopy $n$-sphere and $\theta^{n}$ denotes the group of homotopy $n$-spheres. $H(p, k)$ denotes the subset of ${ }_{\theta}^{p}$ which consists of those homotopy $p$-sphere $\Sigma^{p}$ such that $\Sigma^{p} \times S^{k}$ is diffeomorphic to $S^{p} \times S^{k}$. By [4, Lemma 4], H(p,k) is a subgroup of ${ }_{\theta} p$ and it is not always zero and in fact in [7] we showed that if $k \geq p-3, H(p, k)={ }_{\theta} p$. We shall adopt the notation $E\left(\Sigma^{q}\right)$ to represent the total space of a $p$-sphere bundle over a homotopy $q$-sphere $\Sigma^{q}$. We will then prove the following:

THEOREM. If $M$ is a smooth, $n$-manifold homeomorphic to a $p$-sphere bundle over a $q$-sphere with total space $E$ where $n=p+q \geq 6$ and $p<q$ then there exists homotopy spheres $\Sigma^{q}$ and $\Sigma^{n}$ such that $M$ is diffeomorphic to $E\left(\Sigma^{q}\right) \# \Sigma^{n}$. We shall define a pairing

$$
G: \pi_{p} S O(q) \times \pi_{q-1} S O(p+1) \rightarrow \theta^{p+q}
$$

and show that if $\beta \in \pi_{q-1} S O(p+1)$ is the characteristic class of a p-sphere bundle over an homotopy $q$-sphere $\Sigma^{q}$, then $G\left(\pi_{p} S O(q), \beta\right)$ equals the inertial group of $E\left(\Sigma^{q}\right)$. The above theorem together with the latter will give us the following.

THEOREM. Let $E$ be the total space of a $p$-sphere bundle over a $q$-sphere then the diffeomorphism classes of $(p+q)$-manifolds that are homeomorphic to $E$ are in one-to-one correspondence with the group

$$
\frac{\theta^{q}}{H(p, q)} \times \frac{\theta^{n}}{\text { Image } G_{B}} \text { where } n=p+q \geq 6 \text { and } p<q \text {. }
$$

\section{CLASSIFICATION THEOREM}

In this section, we will prove the classification theorem for any manifold $M^{n}$ homeomorphic to $E$. We will apply the obstruction theory to smoothing of manifolds developed by Munkres in [8]. Since $p+q \geq 6$ and $2 \leq p<q$ then $E$ is simply-connected and the homology of $E$ has no 2-torsion, hence the "Hauptvermutung" of D. Sullivan [9] applies and this means that piecewise linear homeomorphism can be replaced by homeomorphism, we shall not distinguish the two.

DEFINITION. Let $M$ and $N$ be smooth closed $n$-manifolds and $L$ a closed subset of $M$ of dimension less than $n$. Let $f: M \rightarrow N$ be a homeomorphism such that for each simplex $r$ of $L, \bar{\gamma}$ and $f(\bar{\gamma})$ are contained in coordinate systems under which they are flat. $f$ is said to be a diffeomorphism modulo $L$ if $f \mid(M-L)$ is a diffeomorphism and each simplex $\gamma$ of $L$ has a neighborhood $V$ such that $f$ is smooth on $V-L$ near $\gamma$. By [8, Theorem 2.8], if $M$ and $N$ are homeomorphic then there is a diffeomorphism modulo (n-1)-skeleton of $M$. If $f: M \rightarrow N$ is a diffeomorphism modulo m-skeleton $m<n$ then the obstruction to deforming 
$f$ to a diffeomorphism modulo $(m-1)$-skeleton $g: M \rightarrow N$ is an element $\lambda(f) \varepsilon H_{m}\left(M, \Gamma^{n-m}\right)$ where $\Gamma^{n-m}$ is a group of diffeomorphism of $S^{n-m-1}$ modulo those that extend to diffeomorphisms of $D^{n-m}$. $g$ is called the smoothing of $f$. If $\lambda(f)=0$ then by [8, 84$]$ smoothing g exist.

THEOREM 2.1. If $M$ is a smooth $n$-manifold homeomorphic to $E$ where $E$ denotes the total space of a $p$-sphere bundle over a $q$-sphere, $2 \leq p<q$ and $n=p+q$ then there exist homotopy spheres $\Sigma^{q}$ and $\Sigma^{n}$ such that $M$ is diffeomorphic to $E\left(\Sigma^{q}\right) \# \Sigma^{n}$ where $E\left(\Sigma^{q}\right)$ denotes the total space of a p-sphere bundle over the homotopy q-sphere $\Sigma^{q}$.

PROOF. $E$ is the total space of a $p$-sphere bundle over a $q$-sphere with

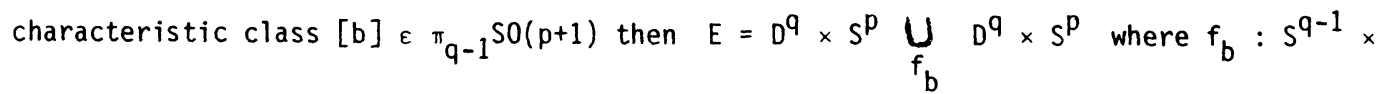
$S^{p} \rightarrow s^{q-1} \times s^{p}$ is a diffeomorphism defined by $f_{b}(x, y)=(x, b(x) \cdot y),(x, y) \varepsilon s^{q-1} \times s^{p}$

$$
H_{j}(E)= \begin{cases}z & \text { for } i=0, p, q, p+q \\ 0 & \text { elsewhere }\end{cases}
$$

Since $M^{n}$ is homeomorphic to $E$ where $n=p+q \geq 62 \leq p<q$, then $M^{n}$ is simply connected and since $\mathrm{H}_{3}(M, Z)$ has no 2-torsion, then "Hauptvermutung" of D. Sullivan [9] implies that there is a piecewise linear homeomorphism $h: M^{n}+E$ which by $[8, \S 5]$ is a diffeomorphism modulo $(n-1)$-skeleton. Since $H_{j}(M, Z)=0$ for $n-p+1 \leq i \leq n-1$ then we can assume that $h$ is a diffeomorphism modulo $n-p=q$ skeleton. The obstruction to a diffeomorphism modulo $q-1$ skeleton is $\lambda(h) \varepsilon H_{q}\left(M, \Gamma^{p}\right)=\Gamma^{p}$. If $[\phi]=\lambda(h) \varepsilon \Gamma^{p}$ where $\phi: s^{p-1} \rightarrow s^{p-1}$ is a diffeomorphism that represents $\lambda(h)$ and let $\Sigma^{p}$ denote the homotopy $p$-sphere where $\Sigma^{p}=D_{1}^{p} \bigcup_{\phi} D_{2}^{p}$. We define a map

$$
j: s^{p}+\Sigma^{p} \quad \text { where } \quad s^{p}=D_{1}^{p} \quad \underset{i d .}{U} D_{2}^{p}
$$

such that

$$
j(x)=\left\{\begin{array}{lllll}
x & \text { if } \quad x & \varepsilon & D_{1}^{p} \\
x & \phi^{-1}\left(\frac{x}{|x|}\right) & \text { if } & x \in & D_{2}^{p} .
\end{array}\right.
$$

So $j$ is an homeomorphism which is identity on $D_{1}^{p}$ and the radial extension of $\phi^{-1}$ on $D_{2}^{p}$ and so the first obstruction $\lambda(j)$ to deforming $j$ to a diffeomorphism is $\left[\phi^{-1}\right]=-\lambda(h)$. We then define id $\times j: D^{q} \times S^{p} \rightarrow D^{q} \times \Sigma^{p}$ where id is the identity, then id $\times j$ is a homeomorphism and it follows from [8, Def. 3.4] that the first obstruction $\lambda(i d \times j)$ to 
deforming id $x j$ to a diffeomorphism is also $-\lambda(h)$. We can form a manifold $E^{\prime}$ by identifying two copies of $D^{q} \times \Sigma^{p}$ along their common boundaries $S^{q-1} \times \Sigma^{p}$ by the diffeomorphism $f_{b}: s^{q-1} \times \Sigma^{p} \rightarrow s^{q-1} \times \Sigma^{p}$ where $f_{b}(x, y)=(x, b(x) y)$ and

[b] $\varepsilon \pi_{q-1} S O(p+1)$. So $E^{\prime}=D^{q} \times \Sigma^{p} \underset{f_{b}}{\bigcup} D^{q} \times \Sigma^{p}$. We define a map

$g: E=\left(D^{q} \times S^{p}\right)_{1}{\underset{f}{f_{b}}}_{U}^{U}\left(D^{q} \times S^{p}\right)_{2} \rightarrow\left(D^{q} \times \Sigma^{p}\right)_{1}{\underset{f}{f_{b}}}_{\bigcup}\left(D^{q} \times \Sigma^{p}\right)_{2}=E^{\prime}$ by $g(x, y)=i d \times j(x, y)$ on both $\left(D^{q} \times \Sigma{ }^{p}\right)_{1}$, and $\left(D^{q} \times S^{p}\right)_{2}$, the map looks like

$$
\begin{aligned}
& E=\left(D^{q} \times S^{p}\right)_{1} \underset{f_{b}}{\bigcup}\left(D^{q} \times S^{p}\right)_{2}=\left(D^{q} \times S^{p}\right)_{1} \underset{f_{b}}{\bigcup} S^{q-1} \times S^{p}{\underset{i d}{U}}_{\left(D^{q} \times S^{p}\right)_{2}}^{\cup} \\
& \lg \quad=\downarrow_{i d \times j} \quad \downarrow_{\text {idx } j} \quad \downarrow_{\text {idxj }} \\
& E^{\prime}=\left(D^{q} \times \Sigma{ }^{p}\right)_{1} \underset{f_{b}}{\bigcup}\left(D^{q} \times \Sigma^{p}\right)_{2}=\left(D^{q} \times \Sigma^{p}\right)_{1} \underset{f_{b}}{\bigcup} s^{q-1} \times S^{p}{\underset{i d}{U}}_{\left(D^{q} \times \Sigma^{p}\right)_{2}}^{U}
\end{aligned}
$$

$g$ is an homeomorphism and the first obstruction to a diffeomorphism is $\lambda(i d x j)=-\lambda(h)$. It follows that the obstructions to smoothing the composition $g \cdot h: M \rightarrow E^{\prime}$ is $\lambda(g \cdot h)=\lambda(g)+\lambda(h)=-\lambda(h)+\lambda(h)=0$. It follows that g.h. $: M \rightarrow E^{\prime}$ is a diffeomorphism modulo $(q-1)$-skeleton. However in [7, Remark 1] we showed that $D^{q} \times \Sigma^{p}$ is diffeomorphic to $D^{q} \times S^{p}$ if $p \leq q+2$ and so by our hypothesis $p<q$ then it follows that $D^{q} \times \Sigma^{p}$ is diffeomorphic to $D^{q} \times S^{p}$. This implies that $E$ and $E^{\prime}$ are diffeomorphic hence $g^{\prime}: M \rightarrow E$ is a diffeomorphism modulo $(q-1)$-skeleton. Since $H_{j}(M, Z)=0$ for $p+1<i<q-1$, there is no more obstruction to deforming $g^{\prime}$ to a diffeomorphism until we get to $(p-1)$ skeleton. We can then assume that $g^{\prime}$ is a diffeomorphism modulo $p$-skeleton. The first obstruction to deforming $g^{\prime}$ to a diffeomorphism modulo $(p-1)-$ skeleton is $\lambda\left(g^{\prime} ;\right) \varepsilon H_{p}\left(M, \Gamma^{q}\right)=\Gamma^{p}$. Let $[\phi]=\lambda\left(g^{\prime}\right) \varepsilon \Gamma^{q}$ where $\phi: s^{q-1} \rightarrow s^{q-1}$ is a diffeomorphism which represents $\lambda\left(g^{\prime}\right) \in \Gamma^{q}$. We define $(\phi \times i d): s^{q-1} \times s^{p} \rightarrow s^{q-1} \times s^{p}$ where $(\phi \times i d)(x, y)=(\phi(x), y)$ and if $\beta=[b] \varepsilon \pi_{q-1} S 0(p+1)$ we also define $f_{b}: s^{q-1} \times s^{p}$ $\rightarrow s^{q-1} \times s^{p}$ where $f_{b}(x, y)=(x, b(x) \cdot y)$. We then have two orientation preserving diffeomorphisms of $s^{q-1} \times s^{p}$ unto itself which we can compose to get $(\phi \times i d) \cdot f_{b}: s^{q-1} \times s^{p} \rightarrow$ $s^{q-1} \times s^{p}$ where $(\phi \times i d) \cdot f_{b}(x, y)=(\phi(x), b(x) \cdot y)$. We then construct a manifold by attaching two copies of $D^{q} \times S^{p}$ along their common boundary $S^{q-1} \times S^{p}$ using the diffeomorphism ( $\times \times i d) \cdot f_{b}$ to have $D_{1}^{q} \times s^{p} \bigcup_{(\phi \times i d) \cdot f_{b}} D_{2}^{q} \times s^{p}$. Notice that this manifold is a p-sphere bundle over a homotopy $q$-sphere $\Sigma^{q}=D_{1}^{q} \bigcup_{\phi} D_{2}^{q}$ whose characteristic map is 
$B=[b] \varepsilon \pi_{q-1} S O(p+1)$. We define a map

by

$$
h: D^{q} \times s^{p} \underset{f_{b}}{U} D_{2}^{q} \times s^{p} \rightarrow D_{1}^{q} \times s^{p} \underset{\text { ( } x i d) \cdot f_{b}}{U} D_{2}^{q} \times s^{p}
$$

$$
h(x, y)= \begin{cases}(x, y) \quad \text { if } \quad(x, y) \in D_{1}^{q} \times s^{p} \\ \left(x \cdot \phi^{-1}\left(\left|\frac{x}{x}\right|\right), y\right) \text { if }(x, y) \in D_{2}^{q} \times s^{p}\end{cases}
$$

Hence $h$ is identity on $D_{1}^{q} \times S^{p}$ and a radial extension of $\phi^{-1}$ on $D_{2}^{q}$. It then follows that $h$ is an homeomorphism with the first obstruction to a diffeomorphism being $\left[\phi^{-1}\right]=$ $-\lambda\left(g^{\prime}\right)$. Then by $[8,3.8]$ the first obstruction to deforming the composition $g^{\prime} \circ h=$ $g: M \rightarrow D_{1}^{q} \times S^{p} \underset{(\phi \times i d) \cdot f_{b}}{U} D_{2}^{q} \times S^{p}$ into a diffeomorphism is $\lambda(g)=\lambda\left(g^{\prime} \circ h\right)=\lambda\left(g^{\prime}\right)+\lambda(h)$ $=-\lambda(h)+\lambda(h)=0$ and hence $g$ is a diffeomorphism modulo $(p-1)$-skeleton. Since $H_{i}(M, Z)=0$ for $0<i<p$ then we can assume that $g$ is a diffeomorphism modulo one point. Since $D_{1}^{q} \times S^{p} \underset{(\phi \times i d) \cdot f_{b}}{U} D_{2}^{q} \times s^{p}$ is a $p$-sphere bundle over a homotopy q-sphere $\Sigma_{1}^{q}=D_{2}^{q} \underset{\phi}{\cup} D^{q}$ with characteristic map $[b] \varepsilon \pi_{q-1} S O(p+1)$, we shall denote it by $E\left(\Sigma^{q}\right)$. Since $g$ is a diffeomorphism modulo one point then it is known that there is an homotopy $n$-sphere $\Sigma^{n}$ such that $M$ is diffeomorphic to $E\left(\Sigma^{q}\right) \# \Sigma^{n}$. Hence the proof.

\section{INERTIAL GROUPS}

Since by Theorem 2.1, every manifold homeomorphic to $E$ is diffeomorphic to $E\left(\Sigma^{q}\right) \# \Sigma^{n}$ for some homotopy spheres $\Sigma^{q}, \Sigma^{n}$, classification of such manifolds reduces to classification of manifolds of the form $E\left(\Sigma^{q}\right) \# \Sigma^{n}$. To complete this classification, we then need to investigate what happens when we vary the homotopy spheres and in particular we need to investigate the Inertial group of $E\left(\Sigma^{q}\right)$. We will investigate these in this section.

LEMMA 3.1. Let $\Sigma_{1}^{q}$ and $\Sigma_{2}^{q}$ be homotopy q-spheres such that $\Sigma_{i}^{q}=D_{1}^{q} \bigcup_{\phi_{i}} D_{2}^{q} i=1,2$ then $E\left(\Sigma_{1}^{q}\right)$ is diffeomorphic to $E\left(\Sigma_{2}^{q}\right)$ if and only if $\Sigma_{1}^{q} \pm \Sigma_{2}^{q} \varepsilon H(q, p)$.

PROOF. Suppose $E\left(\Sigma_{1}^{q}\right)$ is diffeomorphic to $E\left(\Sigma_{2}^{q}\right)$. This means that $D_{1}^{q} \times S^{p} \underset{\left(\phi_{1} \times i d\right) \cdot f_{b}}{U} D_{2} \times S^{p}$ is diffeomorphic to $D_{1}^{q} \times S^{p} \underset{\left(\phi_{2} \times i d\right) \cdot f_{b}}{U} D_{2}^{q} \times S^{p}$ where $\phi_{i} \times$ id $: s^{q-1} \times s^{p} \rightarrow s^{q-1} \times s^{p}$ is the diffeomorphism defined by $\phi_{j}(x, y)=\left(\phi_{j}(x), y\right)$ and $f_{b}: s^{q-1} \times s^{p} \rightarrow s^{q-1} \times s^{p}$ is defined by $f_{b}(x, y)=(x, b(x) \cdot y)$ where $[b]=\beta \varepsilon \pi_{q-1} S O(p+1)$ is the characteristic map of the bundle. The manifold $E\left(\Sigma_{2}^{q}\right)$ can be regarded as the boundary of the $(p+1)$-disc bundle over $\Sigma_{2}$ which is denoted by 
$D_{1}^{q} \times D^{p+1} \bigcup_{\left(\phi_{2} \times i d\right) \cdot f_{b}} D_{2}^{q} \times D^{p+1}=D\left(\Sigma_{2}^{q}\right)$. So if $E\left(\Sigma_{1}^{q}\right)$ is diffeomorphic to $E\left(\Sigma_{2}\right)$ then since $\Sigma_{1}^{q}$ can be embedded in $E\left(\Sigma_{1}^{q}\right)$ it follows that $\Sigma_{1}^{q}$ embedds in $E\left(\Sigma_{2}^{q}\right)$. But $\Sigma_{2}^{q}$ naturally embedds in $E\left(\Sigma_{2}^{q}\right)$ and so we have $\Sigma_{1}^{q}$ and $\Sigma_{2}^{q}$ sitting in $E\left(\Sigma_{2}^{q}\right)$, if we translate $\Sigma_{1}^{q}$ away from $\Sigma_{2}^{q}$ we can run a tube between them to obtain an embedding $\Sigma_{1}^{q} \#\left(-\Sigma_{2}^{q}\right)+E\left(\Sigma_{2}^{q}\right)$ so that the embedding is homotopically trivial and so by the engulfing result of [10, chapter 7] it means that $\Sigma_{1}^{q} \#\left(-\Sigma_{2}^{q}\right)$ can be embedded in the interior of a $(p+q+1)-d i s c$ in $E\left(\Sigma_{2}^{q}\right)$ and by $[11,3.5]$ the embedding is isotopic to a nuclear embedding into the interior of $S^{q} \times D^{p+1}$. However the embedding $\Sigma_{1}^{q} \#\left(-\Sigma_{2}^{q}\right) \rightarrow S^{q} \times D^{p+1}$ is an homotopy equivalence, it then follows by Smale's theorem [12, Theorem 4.1] that $\Sigma_{1}^{q} \#\left(-\Sigma_{2}^{q}\right) \times D^{p+1}$ is diffeomorphic to $S^{q} \times D^{p+1}$ and so it follows that $\Sigma_{1}^{q} \#\left(-\Sigma_{2}^{q}\right) \times S^{p}$ is diffeomorphic to $s^{q} \times S^{p}$ hence $\Sigma_{1}^{q} \#\left(-\Sigma_{2}^{q}\right) \in H(q, p)$. Conversely suppose $\Sigma_{1}^{q} \#\left(-\Sigma_{2}^{q}\right) \in H(q, p)$ then this implies $\left(\Sigma_{1}^{q} \#\left(-\varepsilon_{2}^{q}\right)\right) \times S^{p}$ is diffeomorphic to $S^{q} \times S^{p}$. Since $S^{q} \times S^{p}$ embedds in $R^{p+q+1}$ with trivial normal bundle then it follows that $\Sigma_{1}^{q} \#\left(-\Sigma_{2}^{q}\right)$ embedds in $R^{p+q+1}$ with trivial normal bundle. This shows that each $\Sigma_{i}^{q}$ for $i=1,2$ embedds in $R^{p+q+1}$ with trivial normal bundle and by $[11, \S 3.5]$ the embedding is isotopic to an embedding of $\Sigma_{i}^{q}$ into the interior of $S^{q} \times D^{p+1}$. However for $i=1,2$ the embedding $\Sigma_{i}^{q} \rightarrow S^{q} \times D^{p+1}$ is an homotopy equivalence hence it follows from [12, Theorem 4.1] that $\Sigma_{i}^{q} \times D^{p+1}$ is diffeomorphic to $S^{q} \times D^{p+1}$ which implies that $\Sigma_{1}^{q} \times D^{p+1}$ is diffeomorphic to $\Sigma_{2}^{q} \times D^{p+1}$. Now since $\Sigma_{i}^{q}=D_{1}^{q} \cup D_{2}^{q}$ where $\phi_{i}: s^{q-1} \rightarrow s^{q-1}$ represents $\Sigma_{i}^{q} \in \Gamma^{q} \quad i=1,2$, then we can write $\Sigma_{i}^{q} \times D^{p+1} \stackrel{\dot{i}}{=} D_{1}^{q} \times D^{p+1} \bigcup_{\phi_{i} \times i d} D_{1}^{q} \times D^{p+1}$ where we identify two copies of $D^{q} \times D^{p+1}$ along $S^{q-1} \times D^{p+1}$ by the diffeomorphism $\phi_{j} \times$ id $: S^{q-1} \times D^{p+1} \rightarrow S^{1-1} \times D^{p+1}$ defined by $\left(\phi_{j} \times\right.$ id $)(x, y)=\left(\phi_{j}(x), y\right)$ where $(x, y) \in S^{q-1} \times D^{p+1}$. So $\Sigma_{1}^{q} \times D^{p+1}$ is diffeomorphic to $\Sigma_{2}^{q} \times D^{p+1}$ implies $D_{1}^{q} \times D^{p+1} \bigcup_{\phi_{1} \times i d} D_{2}^{q} \times D^{p+1}$ is diffeomorphic to $D_{1}^{q} \times D^{p+1} \bigcup_{\phi_{2} \times i d} D_{2}^{q} \times D^{p+1}$. Now consider the manifold $D\left(S^{q}\right)=D_{+}^{q} \times D^{p+1} \bigcup_{f_{b}} D_{-}^{q} \times D^{p+1}$ which is a $(p+1)$-disc bundle over a $q$-sphere with characteristic map $[b]{ }_{\varepsilon}^{f} \pi_{q-1} S O(p+1)$. We then form the quotient space

$$
D\left(S^{q}\right) \cup \Sigma^{q}{ }_{1} \times D^{p+1}=\left(D^{q} \times D^{p+1} \bigcup_{f_{b}} \underline{D}^{q} \times D^{p+1}\right) \circlearrowright\left(D_{1}^{q} \times D^{p+1} \bigcup_{\phi_{1} \times i d} D_{2}^{q} \times D^{p+1}\right)
$$

by identifying $D_{-}^{q} \times D^{p+1} \subset D\left(S^{q}\right)$ and $D_{1}^{q} \times D^{p+1} \subset \Sigma_{1}^{q} \times D^{p+1}$ by the relation $(x, y)=$ $(x, y)\left(x \in D_{-}^{q}=D_{1}^{q}, y \in D^{p+1}\right)$. The manifold $D\left(S^{q}\right) \cup \Sigma_{2}^{q} \times D^{p+1}$ is similarly constructed. Since $\Sigma_{1}^{q} \times D^{p+1}$ is diffeomorphic to $\Sigma_{2}^{q} \times D^{p+1}$. Let $d: \Sigma_{1}^{q} \times D^{p+1}+\Sigma_{2}^{q} \times D^{p+1}$ be the 
diffeomorphism and since any diffeomorphism fixes a disc, we can assume that $d$ is identity on the disc $D^{p+q+1}=D_{1}^{q} \times D^{p+1}$, then we can define a diffeomorphism.

$$
g: D\left(S^{q}\right) \cup \Sigma_{1}^{q} \times D^{p+1}+D\left(S^{q}\right) \cup \Sigma_{2}^{q} \times D^{p+1}
$$

where

$$
g(x)=\left\{\begin{array}{lll}
d(x) & \text { for } & x \in \Sigma_{1}^{q} \times D^{p+1} \\
x & \text { for } & x \in D\left(S^{q}\right) .
\end{array}\right.
$$

This means that $g=d$ on $\Sigma_{1}^{q} \times D^{p+1}$ and identity on $D\left(S^{q}\right)$. $g$ is well defined because $d$ is identity on the disc connecting $D\left(S^{q}\right)$ and $\Sigma_{1}^{q} \times D^{p+1}$ and $g$ is a diffeomorphism. The manifold $D\left(S^{q}\right) \cup \Sigma_{i}^{q} \times D^{p+1}$ can be clearly seen as follows. Let $\left(\phi_{i} \times i d\right) \cdot f_{b}: s^{q-1} \times$ ${ }^{D^{p+1}} \rightarrow S^{q-1} \times{ }^{p+1}$ be the diffeomorphism defined by $\left(\left(\phi_{j} \times i d\right) \cdot f_{b}\right)(x, y)=\left(\phi_{j}(x), b(x) \cdot y\right)$, $(x, y) \in S^{q-1} \times D^{p+1}$ then attaching two manifolds $D_{+}^{q} \times D^{p+1}$ and $D_{-}^{q} \times D^{p+1}$ by the diffeomorphism $\left(\phi_{i} i d\right) \cdot f_{b}$ we have $D_{+}^{q} \times D^{p+1} \underset{\left(\phi_{j} \times i d\right) \cdot f_{b}}{U} D_{-}^{q} \times D^{p+1}$ we get a $(p+1)-$ disc bundle over the homotopy $q$-sphere $\Sigma^{q}{ }_{i}=D_{i}^{q} \underset{\phi_{i}}{\cup} D_{2}^{q} \quad i=1,2$. However, from the way $D\left(S^{q}\right) \cup \Sigma_{i}^{q} \times D^{p+1}$ is constructed it is easily seen that $D\left(S^{q}\right) \cup \Sigma_{i}^{q} \times D^{p+1}=$ $D_{+}^{q} \times D^{p+1} \underset{\left(\phi_{i} \times i d\right) \cdot f_{b}}{U} D_{-}^{q} \times D^{p+1}=D\left(\Sigma_{i}^{q}\right)$ hence $g$ is the diffeomorphism of $D\left(\Sigma_{1}^{q}\right)$ onto $D\left(\Sigma_{2}^{q}\right)$ then it follows that $\partial\left(D\left(\Sigma_{1}^{q}\right)\right)=E\left(\Sigma_{1}^{q}\right)$ is diffeomorphic to $\partial D\left(\Sigma_{2}^{q}\right)=E\left(\Sigma_{2}^{2}\right)$.

Hence the theorem is proved.

REMARK 1. This theorem implies that $E\left(\Sigma_{1}^{q}\right)$ is diffeomorphic to $E\left(\Sigma_{2}^{q}\right)$ if and only if $\Sigma_{1}^{q}$ and $\Sigma_{2}^{q}$ are equivalent in the quotient group $\theta^{q} / H(q, p)$.

To complete this classification, we need to determine the inertial group of $E\left(\Sigma^{q}\right)$. The inertial group ${ }_{\perp}(M)$ of an oriented closed smooth $n$-dimensional manifold $M$ is defined to be the subgroup of $\theta^{n}$ consisting of those homotopy $n$-spheres $\Sigma^{n}$ such that $M \# \Sigma^{n}$ diffeomorphic to $M$.

Let $E_{\beta}$ represent the total space of a $p$-sphere bundle over a real q-sphere with characteristic class $B \in \pi_{q-1} S O(p+1)$. In [13] we defined a map $G_{B}: \pi_{p} S O(q) \rightarrow \theta^{p+n}$ and showed that the image of this map equals the inertial group of $E_{\beta}$ where $p<q$ and $E_{\beta}$ has no cross-section. We shall similarly define a map $G_{\phi \cdot \beta}: \pi_{p} S O(q) \rightarrow{ }_{\theta}^{p+q}$ and show that the image of this map equals the inertial group of $E\left(\Sigma^{q}\right)$ where $E\left(\Sigma^{q}\right)$ is the total space of $p$-sphere bundle over a homotopy sphere $\Sigma^{q}=D_{1}^{q} \bigcup_{\phi} D_{2}^{q}$. Let $\alpha \varepsilon \pi_{p} S O(q)$ we define $G_{\phi \cdot \beta}(\alpha)=S^{q-1} \times D^{p+1} \underset{a^{-1}}{ }\left(\bigcup_{\phi \times i d)}^{U} f_{b} D^{q} \times S^{p}\right.$ where $[a]=\alpha$ and $[b]=\beta \varepsilon \pi q-1 S 0(p+1)$ and 
$f_{a-1}(\phi \times i d) \cdot f_{b}: s^{q-1} \times s^{p}+s^{q-1} \times s^{p}$ is a diffeomorphism defined by $f_{a-1}(\phi \times i d) \cdot f_{b}(x, y)$ $=\left(a^{-1}(b(x) \cdot y) \cdot(x), b(x) \cdot y\right)$. One can easily show that $G_{\phi \cdot \beta}$ is well-defined and that its image is an homotopy $(p+q)$-sphere as similarly shown in [13].

LEMMA 3.2. Let $E\left(\Sigma^{q}\right)$ denote the total space of a $p$-sphere bundle over an homotopy q-sphere $\Sigma^{q}=D_{1}^{q}=D_{1}^{q} \cup D_{2}^{q}$ with characteristic class $\beta E \pi_{q-1} S O(p+1)$ then $G_{\phi \cdot B} \pi_{p}(S O(q))=I\left(E\left(\Sigma^{q}\right)\right)$.

PROOF. If $\Sigma^{p+q} \in I\left(E\left(\Sigma^{q}\right)\right)$ then this means there is a diffeomorphism $d: E\left(\Sigma^{q}\right) \# \Sigma^{p+q} \rightarrow E\left(\Sigma^{q}\right)$, that is,

$$
d:\left(D_{1}^{q} \times S^{p} \underset{(\phi \times i d) \cdot f_{b}}{U} D_{2}^{q} \times S^{p}\right) \# \Sigma^{p+q} \rightarrow D_{1}^{q} \times S^{p} \underset{(\phi \times i d) \cdot f_{b}}{U} D_{2} \times S^{p}
$$

since $p<q$ then $\pi_{p}\left(E\left(\Sigma^{q}\right)\right)$ is infinitely cyclic and $d\left(0 \times S^{q}\right)$ represents a generator and so is homotopic to the inclusion $0 \times s^{p} \rightarrow E\left(\Sigma^{q}\right)$. By Haefliger's theorem [14], $d / 0 \times s^{p}$ and the inclusion $0 \times S^{p} \rightarrow E\left(\Sigma^{q}\right)$ are isotopic and by isotopy extension theorem and tubular neighborhood theorem, $d$ is isotopic to a map which we shall again denote by $d$ such that $d \mid D^{q} \times S^{p}=D^{q} \times S^{p}$ where $d(x, y)=(a(y) \cdot x, y)$ for $[a] \varepsilon \pi_{p} S O(q)$ and $(x, y) \in D^{q}$ $\times S^{p}$. We now remove $D^{q} \times S^{p}$ from $E\left(\Sigma^{q}\right) \# \Sigma^{p+q}=\left(D^{q} \times S^{p} \underset{(\phi \times i d) \cdot f_{b}}{U} D^{q} \times S^{p}\right) \# \Sigma^{p+q}$ by surgery away from the connected sum and replace it with $s^{q-1} \times D^{p+1}$. After this operation on the summand $E\left(\Sigma^{q}\right)$ of the connected sum, we have the manifold $S^{q-1} \times D^{p+1}$

$\underset{(\phi \times i d) \cdot f_{b}}{U} D^{q} \times s^{p}$. Since the diffeomorphism $(\phi \times i d) \cdot f_{b}: s^{q-1} \times s^{p}+s^{q-1} \times s^{p}$ extend to the diffeomorphism of $S^{q-1} \times D^{p+1}$ onto itself then $S^{q-1} \times D^{p+1} \underset{(\phi \times i d) \cdot f_{b}}{U} D^{q} \times S^{p}$ is diffeomorphic to $S^{q-1} \times D^{p+1} \bigcup_{i d} D^{q} \times S^{p}$, the diffeomorphism $g$ is defined thus

$$
\left.(\phi \times i d) \cdot f_{b}\right|_{s^{q-1} \times D^{p+1}} \underset{(\phi \times i d) \cdot f_{b}}{U} \underbrace{U}_{D^{q}} \times s^{p}
$$

where

$$
g(x, y)=\left\{\begin{array}{l}
(x, y) \quad \text { if } \quad(x, y) \in D^{q} \times s^{p} \\
\left((\phi \times i d) \cdot f_{b}\right)(x, y) \text { if }(x, y) \in s^{q-1} \times D^{p+1} .
\end{array}\right.
$$

However, by [7, Lemma 2.1.2], $S^{q-1} \times D^{p+1} \bigcup_{i d} D^{q} \times S^{p}$ is diffeomorphic to the standard $(\mathrm{p}+\mathrm{q})$-sphere $\mathrm{s}^{\mathrm{p}+\mathrm{q}}$, hence after this surgery $\mathrm{E}\left(\Sigma^{\mathrm{q}}\right)$ is reduced to $\mathrm{s}^{\mathrm{p}+\mathrm{q}}$ and so $\mathrm{E}\left(\Sigma^{\mathrm{q}}\right) \| \Sigma^{\mathrm{p}+\mathrm{q}}$ is reduced to $\mathrm{s}^{\mathrm{p}+\mathrm{q}} \neq \Sigma^{\mathrm{p}+\mathrm{q}}=\Sigma^{\mathrm{p}+\mathrm{q}}=\Sigma^{\mathrm{p}+\mathrm{q}}$. 
We perform the corresponding modification (under $d$ ) on $E\left(\Sigma^{q}\right)$ to remove the $p$-sohere $0 \times s^{p}$ with product structure $d\left(D_{1}^{q} \times s^{p}\right)$ in $E\left(\Sigma^{q}\right)$. From this modification we obtain a manifold $S^{q-1} \times D^{p+1} \bigcup_{\psi} D^{q} \times S^{p}$ where $\psi=\left(d^{-1} \mid S^{q-1} \times S^{p}\right) \cdot(\phi \times i d) \cdot f_{b}$ and this is diffeomorphic to $\Sigma^{p+q}$ because of the way we performed the surgery using $d$. However, this manifold $S^{q-1} \times D^{p+1} \cup_{\psi} D^{q} \times S^{p}=G_{\phi \cdot \beta}(\alpha)$ by the definition of $G_{\phi \cdot B}$, thus there exists an element $\alpha \varepsilon \pi_{p} S O(q)$ (namely) $d \mid\left(D_{1}^{q} \times S^{p}\right)$ which gives $\alpha \varepsilon \pi_{p} S O(q)$ such that ${ }_{\Sigma}^{p+q}$ $=G_{\phi \cdot \beta}(\alpha)$ and so $\Sigma^{p+q} \varepsilon_{\phi \cdot \beta}\left(\pi_{p} S O(q)\right)$, hence $I\left(E\left(\Sigma^{q}\right)\right) \subset G_{\phi \cdot \beta}\left(\pi_{p} S O(q)\right)$. Conversely suppose $\Sigma^{p+q} \in G_{\phi \cdot \beta}\left(\pi_{p} S O(q)\right)$ then for some $\alpha \varepsilon \pi_{p} S O(q), \Sigma^{p+q}=S^{q-1} \times D^{p+1}$ $f_{a}-1 \cdot(\phi \times i d) \cdot f_{b}$ $D^{q} \times S^{p}$ where $\phi$ is a diffeomorphism of $S^{q-1}$ onto itself representing $\Sigma q=D_{1}^{q} \underset{\phi}{\cup} D_{2}^{q}$ and $f_{a}-1$ and $f_{b}$ are as defined earlier. Notice that $G_{\phi \cdot \beta}(\alpha)$ is thus the obstruction to the construction of a diffeomorphism $S^{p+q}+\Sigma^{p+q}$. To construct a diffeomorphism from $S^{p+q} \rightarrow$ $\Sigma^{p+q}$, we map $s^{q-1} \times D^{p+1} \subset s^{p+q}$ to itself using $(\phi \times i d) \cdot f_{b}$ to have

$$
\begin{aligned}
& s^{p+q}=s^{q-1} \times D^{p+1} \underset{i d}{\cup} D^{q} \times s^{p} \\
& (\phi \times i d) \cdot f_{b} \mid \\
& \Sigma^{p+q}=s^{q-1} \times D^{p+1} \underset{f_{a^{-1}}(\phi \times i d) \cdot f_{b}}{U} D^{q} \times s^{p}
\end{aligned}
$$

and try to extend it to $D^{q} \times S^{p}$. On the boundary $S^{q-1} \times S^{p}$ of $D^{q} \times S^{p}$, the map is

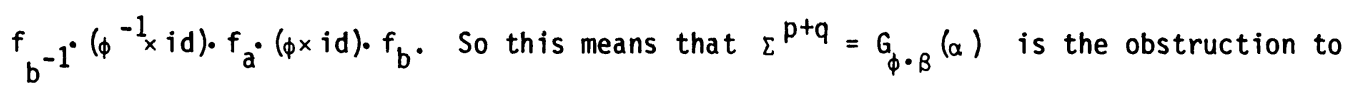
extending the diffeomorphism $f_{b^{-1}}\left(\phi^{-1} \times i d\right) \cdot f_{a} \cdot(\phi \times i d) \cdot f_{b}: s^{q-1} \times s^{p} \rightarrow s^{q-1} \times s^{p}$ to a diffeomorphism of $D^{q} \times S^{p}$ onto itself. We can then define a map $E\left(\Sigma^{p}\right)+E\left(\Sigma^{q}\right)$ using the diffeomorphism $f_{a}: D_{1}^{q} \times S^{p} \rightarrow D_{1}^{q} \times s^{p}$ where $f_{a}(x, y)=(a(y) \cdot x, y)(x, y) \in D_{1}^{q} \times s^{p}$ we then have

$$
\begin{aligned}
& E\left(\Sigma^{q}\right)=D_{1}^{q} \times s^{p} \underset{(\phi \times i d) \cdot f_{b}}{U} D_{2}^{q} \times s^{p} \\
& \downarrow_{a}^{f_{a}} \underbrace{q})=D_{1}^{q} \times s^{p} \bigcup_{(\phi \times i d) \cdot f_{b}} D_{2}^{q} \times s^{p}
\end{aligned}
$$

On the boundary $s^{q-1} \times s^{p}$ of $D_{1}^{q} \times s^{p}$, this map is $f_{b^{-1}}\left(\phi^{-1} \times i d\right) \cdot f_{a}(\phi \times i d) \cdot f_{b}$ and the obstruction to extending this to a diffeomorphism of $E\left(\Sigma^{q}\right)$ onto itself is the 
obstruction to extending the map $f_{b}-1 \cdot\left(\phi^{-1} \times i d\right) \cdot f_{a} \cdot(\phi \times i d) \cdot f_{b}$ to the diffeomorphism of $D_{2}^{q} \times s^{p}$ onto itself which is $\Sigma^{p+q}$. It then follows that $E\left(\Sigma^{q}\right)+E\left(\Sigma^{q}\right) \# \Sigma^{p+q}$ is a diffeomorphism and so $\Sigma^{p+q} \varepsilon I\left(E\left(\Sigma^{q}\right)\right)$ hence

$$
E\left(E\left(\Sigma^{q}\right)\right)=G_{\phi \cdot \beta} \pi_{p}(S O(q)
$$

REMARK 2. We note that if $p=2,4,5,6(\bmod 8)$ and $p<q-1$ then $\pi_{p} S O(q)=0$ and so the image of $G$ is trivial and hence in this particular case, the inertial group of $E\left(\Sigma^{q}\right)$ is trivial and this coincides with the result of [4, Proposition 1].

REMARK 3. By [15], intertial group $I(M)$ of a smooth manifold $M$ is a diffeotopy invariant of $M$. So if $2 p \geq q+1$ then we can deduce that the inertial group $I\left(E\left(\Sigma^{q}\right)\right)$ of a $p$-sphere bundle over an homotopy $q$-sphere $\Sigma^{q}$ is equal to the inertial group $I\left(E_{\beta}\right)$ of a $p$-sphere bundle over the standard $q$-sphere, where $\beta \varepsilon \pi_{q-1} S O(p+1)$ classifies the associated disc bundle. Let $D\left(\Sigma^{q}\right)$ be the associated $(p+1)$-disc bundle over the homotopy q-sphere where $E\left(\Sigma^{q}\right)$ is the boundary of $D\left(\Sigma^{q}\right)$. $\Sigma^{q}$ has the homotopy type of $D\left(\Sigma^{q}\right)$ and $\Sigma^{q}$ has the homotopy type of $S^{q}$, it follows that $s^{q}$ has the homotopy type of $D\left(\Sigma^{q}\right)$. Since $2 p \geq q+1$ then it follows that $2(p+q+1) \geq 3 q+3$ and since $p+q>5$ and $p \geq 3$ then $D\left(\Sigma^{q}\right)$ and $E\left(\Sigma^{q}\right)$ are simply connected and from [12: Theorem 4.4], it follows that $D\left(\Sigma^{q}\right)$ is diffeomorphic to a $(p+1)$-disc bundle $D\left(s^{q}\right)$ over the $q$-sphere $s^{q}$ hence the boundary $\partial D\left(\Sigma^{q}\right)=E\left(\Sigma^{q}\right)$ of $D\left(\Sigma^{q}\right)$ is diffeomorphic to the boundary $\partial D\left(S^{q}\right)=E_{B}$ of $D\left(S^{q}\right)$. It then follows by [15] that $I\left(E\left(\Sigma^{q}\right)\right)=I\left(E_{\beta}\right)$. This means that the inertial group of $S_{\beta}$ in [13] coincides with Lemma 3.2 .

Combination of Lemmas 3.1 and 3.2 give the following.

THEOREM 3.3. Let $E$ be the total space of a $p$-sphere bundle over a $q$-sphere with characteristic map $\beta \in \pi_{q-1} S O(p+1)$ then the diffeomorphism classes of $p+q-$ manifolds that are homeomorphic to $E$ are in one-to-one correspondence with the group

$$
\frac{\theta^{q}}{H(q, p)} \times \frac{\theta^{n}}{\text { Image } G_{B}}
$$

where $p+q=n \geq 6$ and $p<q$.

\section{REFERENCES}

1. De Sapio, R., Manifolds Homeomorphic to Sphere Bundles Over Spheres. Bull. American Math. Soc. 75 (1969), 59-63.

2. Munkres, J., Concordance of Differentiable Structures - Two Approaches. Michigan Math. J. 14 (1967), 183-191. 
3. Schultz, R., Smoothing of Sphere Bundles of Spheres in Stable Range. Inventiones Math 9 (1969), 81-88.

4. De Sapio, R., Differential Structures on a Product of Spheres. Comm. Math. Helv. Vol. $44, \underline{1}$ (1969), 61-69.

5. Kawakubo, K., Smooth Structures on $S^{p} \times S^{q}$. Proc. Japan Acad. 45 (1969), 215-218.

6. Schultz, R., Smooth Structures on $S^{p} \times S^{q}$. Annals of Math. 90 (1969), 187-198.

7. Ajala, S. 0., Differentiable Structures on Product of Spheres. Houston Journal of Math., Vol. 10, 1 (1984), 1-14.

8. Munkres, J., Obstruction to Smoothing of Piecewise-Differentiable Homeomorphisms. Annals of Math. Vol. 72, $\underline{3}$ (1960), 521-554.

9. Sullivan, D., On Hauptvanmutung for Manifolds. Bull. Amer. Math. Soc. 73 (1967), 598-600.

10. Zeeman, E., Seminar on Combinatorial Topology (mimeogrpahed notes), Inst. Hautes Etudes, Sci. Publ. Math. (1965).

11. Levine, J., Classification of Differentiable Knots. Annals of Math. 82 (1965), 15-50.

12. Smale, S., On the Structure of Manifolds. Amer. Math. J. 84 (1962), 387-399.

13. Ajala, S. 0., Inertial Group of $p$-sphere Bundle over a $q$-sphere without Cross-section. Nigerian Journal of Science Vol. 18, (1984), to appear.

14. Haefliger, A., Plongements Differentiables de Varietes dans Varietes, Comm. Math. Helv. 36 (1961), 47-81.

15. Kawakubo, K., Inertial Group of Homology Tori. J. Math. Soc. Japan Vol. 21 , 1 (1969).

16. Hsiang, W. C., Leving, J., and Szczarba, R. H., On the Normal Bundle of a Homotopy Sphere Embedded in Euclidean Space. Topology 3 (1965), 173-181. 


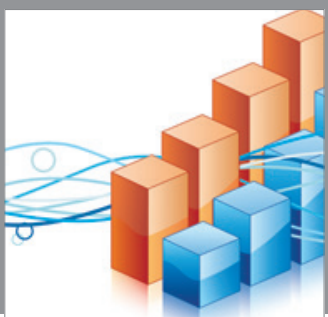

Advances in

Operations Research

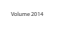

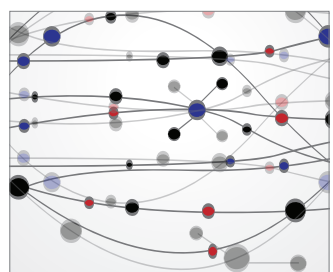

\section{The Scientific} World Journal
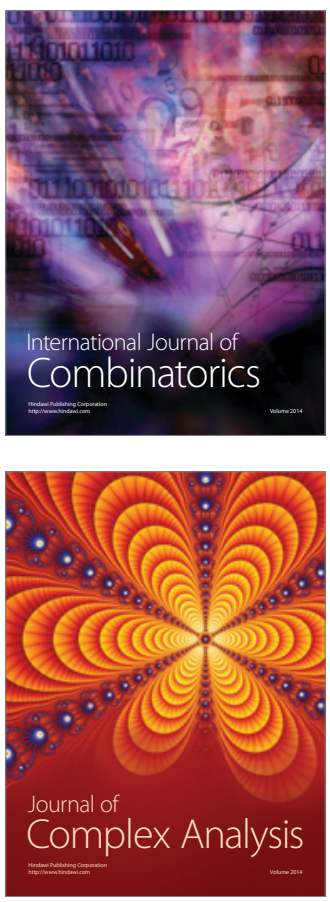

International Journal of

Mathematics and

Mathematical

Sciences
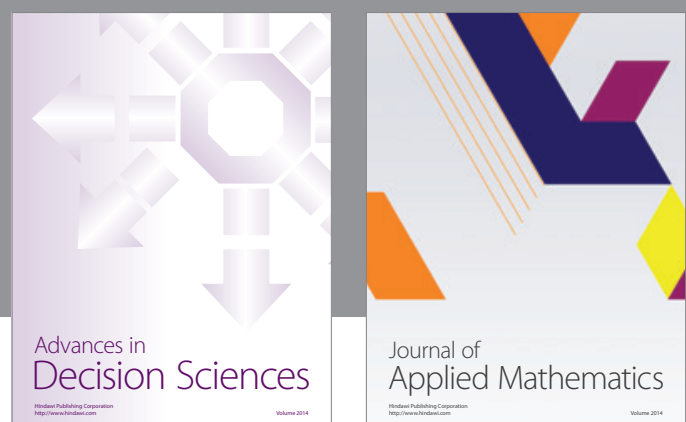

Journal of

Applied Mathematics
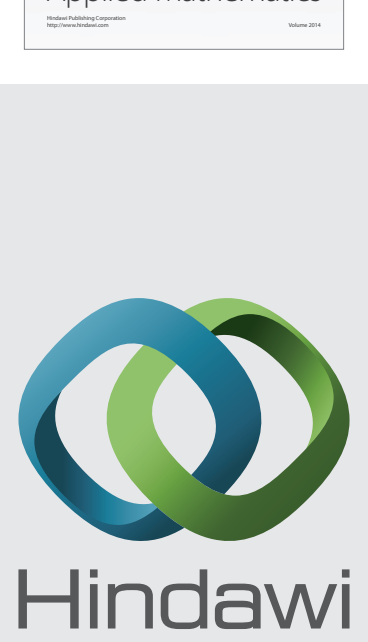

Submit your manuscripts at http://www.hindawi.com
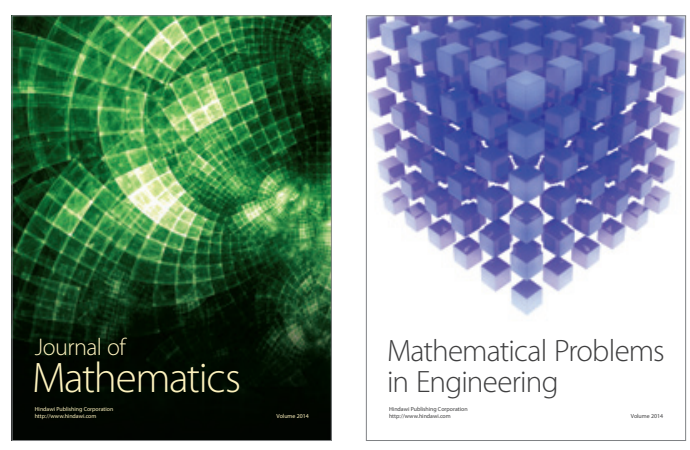

Mathematical Problems in Engineering
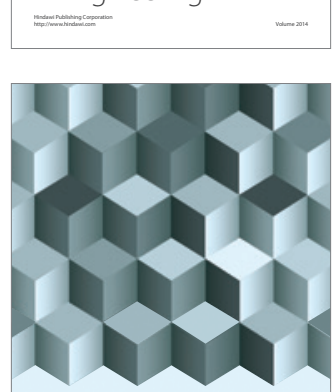

Journal of

Function Spaces
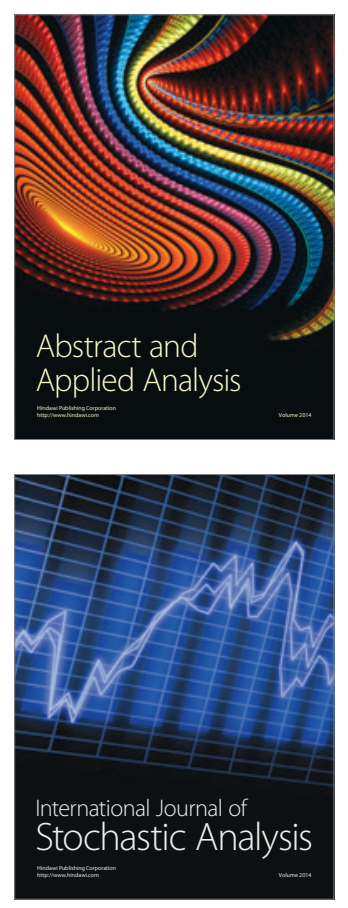

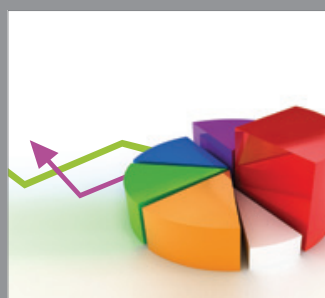

ournal of

Probability and Statistics

Promensencen
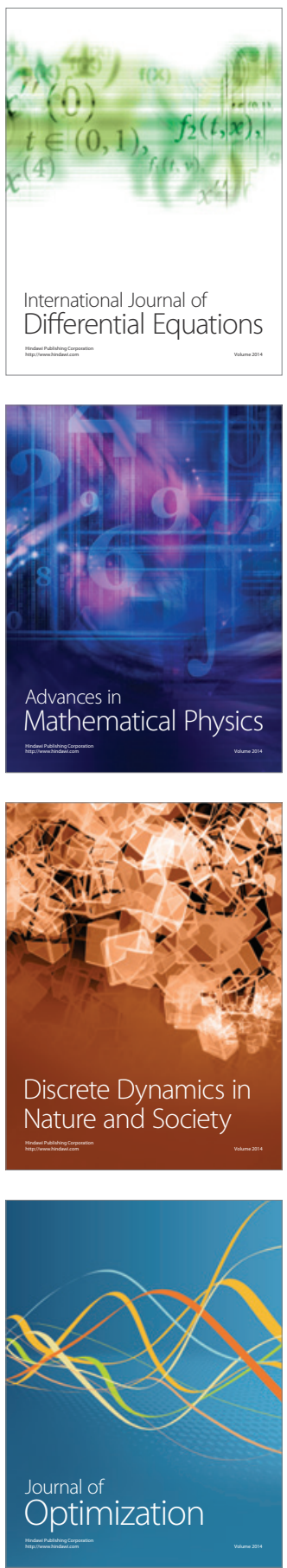